Results A representative sample of 520 individuals participated; average age was 36.8 years (SD 9.9), 40\% were males and $66 \%$ had a Bachelor's degree or higher. The choice to test was most influenced by cost (free), followed by speed of results (in 14 days), delivery of results (via SMS), extra STI testing, appointment available (same day), and the least important was location of testing (private hospital). The choice to attend for management of chlamydia was most influenced by the attitude of staff (not rude), followed by cost (free), who they consult (specialist), access to PDPT, travel time (less than 30 minutes) and the least important was treatment location (antibiotics sent to home). There was significant heterogeneity in preferences related to age, place of birth, those reporting more than one sexual partner in the preceding six months and gender.

Conclusion To design effective chlamydia testing and management services, it is important to account for patient preferences. For people living in Hong Kong, cost and staff attitude were the most important factors for deciding whether to test or be managed for chlamydia, respectively.

\section{P311 ASSOCIATION BETWEEN CHOICE OF NON-BARRIER CONTRACEPTIVE AND CONDOM USE AMONG FSWS IN CHINA: IMPLICATIONS FROM A NATIONAL CROSS- SECTIONAL STUDY}

1,2,3Y Wang*, ${ }^{4} \mathrm{~K}$ Dooley, ${ }^{5} \mathrm{~W}$ Zhang, ${ }^{4} \mathrm{~K}$ Smith, ${ }^{1,2,3} \mathrm{C}$ Wang. ${ }^{1}$ Dermatology Hospital of Southern Medical University, Guangzhou, China; ${ }^{2}$ Southern Medical University Institute for Global Health and Sexually Transmitted Diseases, Guangzhou, China; ${ }^{3}$ Guangdong Provincial Center for Skin Disease and STI Control, Guangzhou, China; ${ }^{4}$ Division of Epidemiology and Community Health, University of Minnesota Twin Cities, Minneapolis, USA; ${ }^{5}$ University of North Carolina at Chapel Hill, Project-China, Guangzhou, China

\subsection{6/sextrans-2021-sti.366}

Background Condom promotion was primarily to prevent human immunodeficiency virus (HIV) among Chinese female sex workers (FSWs), which may have missed to improve their reproductive health and sexual health holistically. A more complete understanding of FSWs' sexual and reproductive health needs specifically pertaining to the choice of contraceptive would be beneficial to make progress in disease control. This study aimed to better characterize contraceptive use and explore the interaction between contraception and condom use among Chinese FSWs.

Methods A cross-sectional study was conducted in eight cities throughout 7 provinces in China, 2019. Participants completed a survey including sociodemographic information, sexual behaviors and reproduction measures. The exposure of interest was non-barrier contraceptive use, and the outcome of interest was inconsistent condom use with clients. Multivariate logistic regression and subgroup analysis were conducted to assess the relationship between non-barrier contraceptive use and condom use among Chinese FSWs.

Results In total, 1229 FSWs participated in the study with a median age of 35 years. 629(51.2\%) women reported using non-barrier contraceptives while 586 (47.7\%) used barrier contraceptives. Odds of inconsistent condom use was higher $(\mathrm{aOR}=1.24$, 95\% CI: 0.99-1.57) among nonbarrier contraceptives users than among barrier contraceptives users, but it lacks statistical significance. However, for women in middle-tier venues $(\mathrm{aOR}=0.66,95 \% \mathrm{CI}$ : 0.46-0.96), upper-tier venues $(\mathrm{aOR}=1.64$, 95\% CI: 1.14 2.36), and those who completed senior high school or above (aOR $=4.07,95 \%$ CI: 2.20-7.52), we observed correlation between inconsistent condom use and non-barrier contraception.

Conclusion We observed that non-barrier contraceptive users who work in upper tier settings and with higher educational attainment tend to use condom inconsistently. Condom promotion efforts currently focused on HIV and STI prevention among FSWs in China would benefit from considering the contraceptive and reproductive needs of this population.

\section{P312 DESIGNING HIV TESTING AND SELF-TESTING SERVICES FOR YOUNG PEOPLE IN NIGERIA: A DISCRETE CHOICE EXPERIMENT}

${ }^{1} \mathrm{~J}$ Ong ${ }^{*},{ }^{2} \mathrm{U}$ Nwaozuru, ${ }^{2} \mathrm{C}$ Obiezu-Umeh, ${ }^{3} \mathrm{C}$ Airhihenbuwa, ${ }^{2} \mathrm{H}$ Xian, ${ }^{4} \mathrm{~F}$ Terris-Presholt, ${ }^{5} \mathrm{~T}$ Gbajabiamila, ${ }^{5} \mathrm{D}$ Oladele, ${ }^{5} \mathrm{I}$ Idigbe, ${ }^{5} \mathrm{~A}$ David, ${ }^{5} \mathrm{~J}$ Okwuzu, ${ }^{5} \mathrm{~T}$ Bamidele, ${ }^{2} \mathrm{~J}$ Iwelunmor, ${ }^{6}$ J Tucker, ${ }^{5} 0$ Ezechi. ${ }^{1}$ Melbourne Sexual Health Centre; ${ }^{5} 80$ Swanston Street, Australia; ${ }^{2}$ Saint Louis University College for Public Health and Social Justice, USA; ${ }^{3}$ Georgia State University, Atlanta, USA; ${ }^{4}$ UNAIDS, Switzerland; ${ }^{5}$ The Nigerian Institute of Medical Research, Lagos, Nigeria; ${ }^{6}$ London School of Hygiene and Tropical Medicine, London, UK

\subsection{6/sextrans-2021-sti.367}

Introduction A third of new HIV infections occur among young people and the majority of young people living with HIV are in sub-Saharan Africa. Global scale-up of HIV selftesting (HIVST) could improve testing rates among young people in SSA, who have reported suboptimal HIV testing. We examined the strength of Nigerian youth preferences related to HIV testing.

Methods Discrete choice experiments (DCEs) were conducted among Nigerian youth (age 14-24 years). Participants completed one of two DCEs: one for preferred qualities of HIV testing (cost, location of the test, type of test, the person who conducts the test, and availability of HIV medicine at the testing site) and another for preferred qualities of HIVST kits (cost, test quality, type of test, extra items and support if tested positive)

We use random parameters logit model to quantify the relative strength of preferences and test for preference heterogeneity.

Results A total of 504 youth participated: mean age 21 (SD 2) years, $38 \%$ were men, and $35 \%$ had higher than secondary school education. Youth equally preferred HIV testing services in public hospitals and home testing, all other attributes being equal. For HIVST kits, youth preferred the kit to be low cost (up to \$USD1.38), accessible from community health centers, and integrated with self-testing for syphilis and other sexually transmitted infections (STIs). Preferences differed according to age, education level, gender, sexual, and HIV testing behaviours.

Conclusions We found that there could be demand for HIVST for Nigerian youth, who preferred HIVST kits that integrate testing for other STIs and is accessed from community health centers. These data could inform tailoring of HIV testing delivery services for Nigerian youth, to ultimately increase reach and acceptability. 